\title{
Dezocine: a novel drug to prevent fentanyl-induced cough during general anesthesia induction?
}

\author{
Qiang Sun $\cdot$ Wei Zhou $\cdot$ Bo Wu $\cdot$ Mu-huo Ji $\cdot$ \\ Yong G. Peng
}

Received: 31 October 2011 / Accepted: 19 December 2011/Published online: 7 January 2012

(C) Japanese Society of Anesthesiologists 2012

To the Editor:

We read with great interest the recently published article by Dr. Sun et al. [1], in which the authors demonstrated that intravenous administration of $0.1 \mathrm{mg} / \mathrm{kg}$ dezocine $10 \mathrm{~min}$ before general anesthesia completely suppressed fentanyl-induced cough during induction. The results were very inspirational and astonishing. However, we note that standard dose and usage of fentanyl do not cause a significant problem in clinical practice. Furthermore, we find that there is a clerical error in this article. The authors suggested that dezocine antagonizes $\kappa$ receptors, which in turn antagonize fentanyl-activated $\mu$ receptors, thereby inducing cough. This statement was in disagreement with the results of their article, in which they showed dezocine was effective in suppressing fentanyl-induced cough during general anesthesia induction. From this point of view, it should be restated that dezocine antagonizes $\kappa$ receptors, which in turn antagonize fentanyl-activated $\mu$ receptors, thereby reducing cough.

In addition, the mechanisms of fentanyl-induced cough have been multifactorial including opioid receptors, C-fiber receptors, rapidly adapting pulmonary stretch receptors,

Q. Sun · B. Wu

Department of Anesthesiology, Institution of Stomatology, College of Stomatology, Nanjing Medical University, Nanjing, People's Republic of China

W. Zhou · M. Ji ( $\square)$

Department of Anesthesiology, Jinling Hospital, School

of Medicine, Nanjing University, 305 East Zhongshan Road,

Nanjing 210002, People's Republic of China

e-mail: ufo5201981@gmail.com

Y. G. Peng

Department of Anesthesiology, University of Florida College

of Medicine, Gainesville, FL, USA histamine, and the citrate in the fentanyl injection fluid, just as the authors suggested [1]. However, the authors provided inadequate evidence to support their claim that dezocine counteracted the action of fentanyl by a central gating mechanism of cough suppression, predominantly via C-fiber receptors, or, whether dezocine inhibited the release of histamine by antagonizing and suppressing cough reflex? These speculations may have overextrapolated their study results because there is no evidence supporting the mechanisms of dezocine involved in the suppression of fentanyl-induced cough during general anesthesia induction. Therefore, more studies are needed to clarify the effects and the precise mechanisms of dezocine involved in suppressing fentanyl-induced cough during general anesthesia induction.

Conflict of interest The authors have no potential conflicts of interest to disclose.

\section{Reference}

1. Sun ZT, Yang CY, Cui Z, Zhang J, Han XP. Effect of intravenous dezocine on fentanyl-induced cough during general anesthesia induction: a double-blinded, prospective, randomized, controlled trial. J Anesth 2011 [Epub ahead of print]. 